\title{
Magnetic resonance-guided direct shoulder arthrography for the detection of superior labrum anterior-posterior lesions using an open 1.0-T MRI scanner
}

\author{
Alexander Berth ${ }^{1 A, B, C, D, E, F, G}$, Agnieszka Pozniak ${ }^{1 E, F}$, Linda Stendel ${ }^{2 B, F}$, Frank Fischbach ${ }^{3 A, B, C, D, E}$, \\ Christoph Lohmann $3 \mathrm{~A}, \mathrm{D}, \mathrm{E}, \mathrm{G}$, Maciej Pech ${ }^{3 \mathrm{~A}}$ \\ 'Department of Orthopaedic Surgery, Otto-von-Guericke-University, Magdeburg, Germany \\ 2Department of Internal Medicine, Asklepios Clinic Hamburg Barmbek, Germany \\ ${ }^{3}$ Department of Radiology and Nuclear Medicine, Otto-von-Guericke University, Magdeburg, Germany
}

\section{Abstract}

\begin{abstract}
Purpose: Direct magnetic resonance arthrography (MRA) offers increased diagnostic accuracy compared to conventional magnetic resonance imaging (MRI) in the detection of superior labrum anterior-posterior (SLAP) lesions. The aim of the present study was to present the technique of magnetic resonance-guided direct shoulder arthrography (MDSA), to evaluate the diagnostic value of this novel MRA procedure to detect SLAP lesions in comparison to the currently practiced MRI, and to correlate the radiological findings to the respective arthroscopic findings.

Material and methods: Fifty-six patients with clinical signs of a SLAP lesion underwent both MRI examination and MDSA prior to arthroscopic surgery. The MRI of both interventions were compared with the arthroscopic findings. Statistical analysis was performed using the McNemar test.

Results: Sensitivity, specificity, and accuracy for detecting SLAP lesions were $23 \%, 88 \%$, and $54 \%$ on MRI and $80 \%$, $81 \%$, and $80 \%$ on MDSA, respectively. Sensitivity $(p<0.001)$ and accuracy $(p=0.001)$ in detection of SLAP lesions were significantly higher by MDSA whereas accuracy showed no significant differences $(p=0.625)$.

Conclusions: The MDSA can be performed in an open 1.0-T MRI scanner with a high level of technical success and a reasonable methodical effort. The modification of MRA provides the requirements as a practicable routine shoulder magnetic resonance examination including arthrography to detect SLAP lesions. The diagnostic value is significantly better than MRI examinations without included arthrography, which currently predominates the clinical practice to investigate shoulder pathology.
\end{abstract}

Key words: SLAP lesions, shoulder, MR arthrography, conventional MRI, shoulder arthroscopy.

\section{Introduction}

Pathologies of the capsulolabral complex such as superior labrum anterior-posterior (SLAP) lesions are often the reason for shoulder pain and instability [1]. Various traumatic mechanisms exist, which may lead to these labral tears [2-6]. According to Snyder et al. [3], they mostly occur after a fall on an outstretched arm with the compression of the humeral head against the superior labrum. Another reason identified may be high traction forces of the arm that cause the overstretching and lesions of the superior capsulolabral complex. The diagnostic assessment of SLAP lesions by conventional magnetic resonance imaging (MRI) seems to be the most frequent method in daily practice, although the diagnostic reliability of this method has great variability [7]. One possible explanation for these

Correspondence address:

Alexander Berth, MD, PhD, Department of Orthopaedic Surgery, Otto-von-Guericke-University Magdeburg, 44 Leipziger St., 39120 Magdeburg, Germany,

e-mail: alexander.berth@med.ovgu.de

Authors' contribution:

A Study design · B Data collection · C Statistical analysis · D Data interpretation · E Manuscript preparation · F Literature search · G Funds collection 


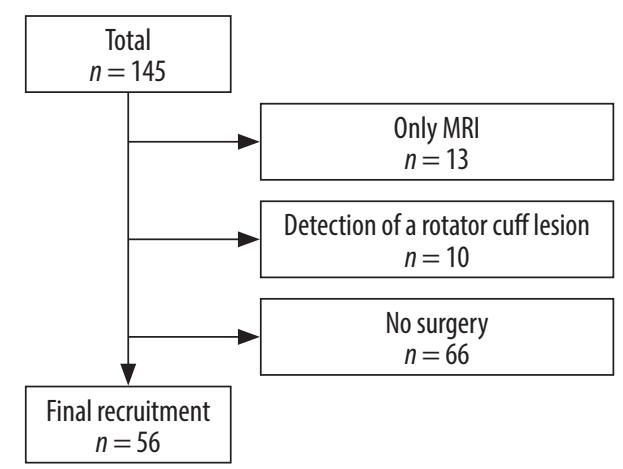

Figure 1. Study group recruitment

different results might be that the superior capsulolabral complex demonstrates numerous physiological variations. This increases the difficulty in discriminating between the mentioned physiological variations and pathological changes due to degenerative and/or traumatic causes of the superior capsulolabral complex $[8,9]$.

As an alternative to MRI, direct magnetic resonance arthrography (MRA) can be used in the detection of SLAP lesions. In contrast to conventional MRI, the contrast medium can be applied into the joint during MRA. Due to the inflation of the joint capsule, MRA gives a better possibility to distinguish the anatomical structures. The diagnostic reliability of MRA is more accurate [10-12]. However, despite these data, MRA is not established as a standard procedure in the diagnostics of SLAP lesions due to the methodological aspects of this method.

The aim of this study was to present a novel method of MR-assisted shoulder arthrography (MDSA), representing a modification of MRA, considering the requirements of a practicable procedure in the daily practice diagnosing suspected SLAP lesions. Furthermore, we compared

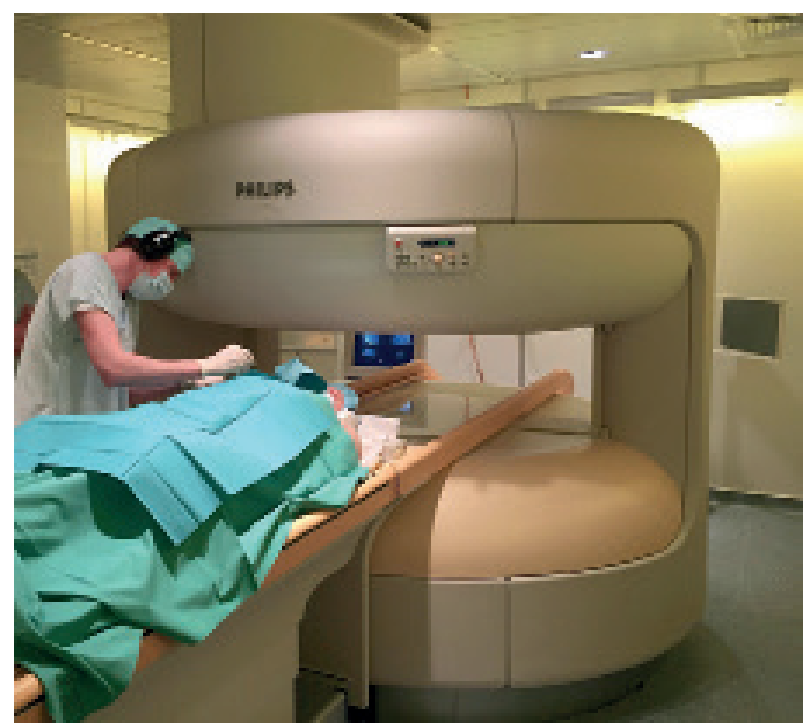

Figure 2. The open 1.0-T MRI scanner with two parallel superconducting magnet poles. The open configuration allows a direct intra-articular injection of the contrast medium. No repositioning of the patient or changing of the receiver coil was necessary the diagnostic findings with those of conventional MRI investigations with respect to the diagnostic reliability of this method for the detection of SLAP lesions, using the findings in arthroscopic surgery as a reference.

We posed the hypothesis that MDSA leads to more reliable and accurate diagnostic accuracy compared to MRI.

Therefore, we used 56 patients and performed conventional MRI as well as MDSA and correlated the radiological findings with surgical results.

\section{Material and methods}

\section{Patients and recruitment protocol}

This prospective cohort study included 145 patients recruited in the period from December 2012 until June 2016. The inclusion criteria were patients who presented a symptomatic SLAP lesion, aged between 20 and 45 years, and symptomatic for at least three months. Due to a persistent pain, loss of shoulder function despite conservative treatment, and the clinical suspicion for a SLAP lesion, patients underwent a magnetic resonance examination according to the following study protocol. Patients with previous shoulder surgery on the affected joint and those with concomitant pathologies confirmed in the MR diagnostic were excluded.

For various reasons (e.g. claustrophobia) in 13 cases only a conventional MRI could be performed. Based on the request of 66 patients who were examined with both MRI and MDSA, the decision was made to continue the conservative treatment. In 10 patients the MRI investigations demonstrated evidence of a rotator cuff lesion, and therefore these patients were excluded from the study. Finally, there were 56 patients ( 38 males / 18 females, age $26 \pm 16$ years) in the cohort. Finally, the indication for operative arthroscopic treatment in these 56 was based on a persistent, severe or moderate pain and loss of shoulder function despite conservative treatment, and radiological evidence for a SLAP lesion by MRI and MDSA investigation (Figure 1).

The study was performed in accordance with the ethical standards of the 1964 Declaration of Helsinki and was approved by the local Ethical Committee (Reg. No. 84/11). Written, informed consent was obtained from all patients.

\section{Magnetic resonance tomography}

MRI and MDSA were performed using an open MRI scanner - Panorama HFO (Philips Healthcare, Best, Netherlands). This MR scanner has a vertical static magnetic field of $1.0 \mathrm{~T}$ created by two horizontally opposed superconducting magnet pole shoes that provide a maximum gradient strength of $26 \mathrm{mT} / \mathrm{m}$ and a slew rate of $80 \mathrm{~T} / \mathrm{m} / \mathrm{s}$. A dedicated shoulder coil was used for signal reception during MRI and MDSA investigations. The patients were placed in a supine position with slight external rotation of the adducted arm (Figure 2). 
The standard protocol comprised T1-, T2-, and $\mathrm{PD}$-weighted images in the oblique coronal, oblique sagittal, and transverse plane.

The following parameters for the MR sequences were defined:

- T1 TSE imaging (TR: $675 \mathrm{~ms} / \mathrm{TE} 10 \mathrm{~ms}$ in transverse, adjusted coronal),

- T2 TSE imaging (TR: $3000 \mathrm{~ms} / \mathrm{TE} 90 \mathrm{~ms}$ in adjusted sagittal),

- $\quad$ PD TSE imaging fat-saturated (TR $2250 \mathrm{~ms} / \mathrm{TE} 30 \mathrm{~ms}$ in transverse, adjusted coronal, and sagittal).

The resolution was by measured Voxel size with edge length of $0.71 \times 0.5 \mathrm{~mm}$ for PD-weighted and T2-weighted imaging and $0.6 \times 0.9 \mathrm{~mm}$ for T1-weighted imaging. For all measurements the slice thickness was $2.5 \mathrm{~mm}$.

In all the patients the MSDA investigation followed immediately after the conventional MRI. The technique of puncture of the shoulder joint and intra-articular injection of the contrast medium was done according to the study of Wybranski et al. [13]. After antiseptic skin preparation, sterile draping, and administration of local anaesthetics the puncture needle trajectory and position were controlled under constant MR fluoroscopy with a $22 \mathrm{G}$ MRI-compatible needle, and $20 \mathrm{ml}$ of $2.5 \mathrm{mmol} / \mathrm{l}$ gadoteric acid solution (Artirem ${ }^{\oplus}$, Guebet, Roissy, France) was injected. After removal of the puncture needle the MR imaging was repeated.

\section{Surgical technique}

All operations were performed by the senior author with the patients in a beach chair position under general anaesthesia in combination with an interscalenary block. The patient's arm was placed in an articulated hydraulic arm holder (Trimano ${ }^{\oplus}$, Arthrex, Naples, USA) for various positions. Three routine arthroscopic portals (anterior, lateral, posterior) were used during the procedure if necessary additional portals (e.g. posterolateral, transtendinous) were created to perform the SLAP repair.

\section{Evaluation}

Based on the arthroscopic findings, the configurations of superior capsulolabral complex were described and classified according to Snyder [3].

For MDSA, the technical success rate (defined as intraarticular contrast medium injection) and the time for the application of the contrast medium (starting point: beginning of the skin preparation, end: removal of the injection needle) were also measured.

MRA images were reviewed by an experienced radiologist who was blinded to the patients' histories and arthroscopic findings as well as definite diagnoses. The conventional MRI and MDSA images were separately analysed.

All MR diagnoses were compared to the reports of the shoulder arthroscopies, which served as references.
The mean time from MRI examinations and shoulder arthroscopies was approximately $78 \pm 70$ days (min: 4 days, max: 297 days).

\section{Statistics}

The diagnostic performance of MRI and MDSA in the diagnosis of arthroscopic proved that SLAP lesions are analysed for sensitivity, specificity, and accuracy.

The estimation of an appropriate sample size was calculated using the ROCPOWER/SAS ${ }^{\oplus}$ program with a cut-off of AUC (area under the curve) of ROC (receiver operating characteristics) analysis.

The McNemar test was used to compare the diagnostic values of both MRI investigations.

A significance level less than 0.05 was assumed. SPSS statistical software, version 18.0, was used for all calculations. Unless specified otherwise, results are given as mean \pm standard deviation.

\section{Results}

The overall technical success rate for MDSA was $100 \%$ (correct intra-articular injection, no periarticular leakage). No injuries or adverse events, such as bleeding or allergic reaction to the contrast medium, were observed. The average time needed for preparing and performing the MR-guided arthrography was 10.8 minutes (minimum: 9.9 minutes, maximum: 11.7 minutes).

Shoulder arthroscopy indicated the presence of SLAP lesions in 29 of the 56 patients (52\%). According to the reference standard 6 patients $(21 \%)$ had a type I lesion, 22 patients had a type II lesion (76\%) and 1 patient (3\%) had a type IV lesion. There were no type II SLAP lesions in the study group. In the detection of SLAP lesions with conventional MRI, there were seven true positive, 23 false negative, and three false positive results. In the assessment of the superior capsulolabral complex with regard to SLAP lesions with the MDSA there were 24 true positive, six false negative, and five false positive results. The results of the diagnostic efficacy of MRI and MDSA in the diagnosis of SLAP lesions are presented in Table 1.

Figure 3 demonstrates MRI and MDSA scans in coronary view showing an intact superior capsulolabral complex and the corresponding arthroscopic finding. Coronal scans and (B) axial fat-suppressed T1-weighted MR arthro-

Table 1. Results of calculated values for sensitivity and specificity of conventional magnetic resonance imaging (MRI) and magnetic resoananceassisted shoulder arthrography (MDSA) compared with arthroscopy as the reference method

\begin{tabular}{|l|c|c|c|} 
& MRI & MDSA & $p$-value \\
\hline Sensitivity & $23 \%$ & $80 \%$ & $<0.001$ \\
\hline Specificity & $88 \%$ & $81 \%$ & 0.625 \\
\hline Accuracy & $54 \%$ & $80 \%$ & 0.001 \\
\hline
\end{tabular}



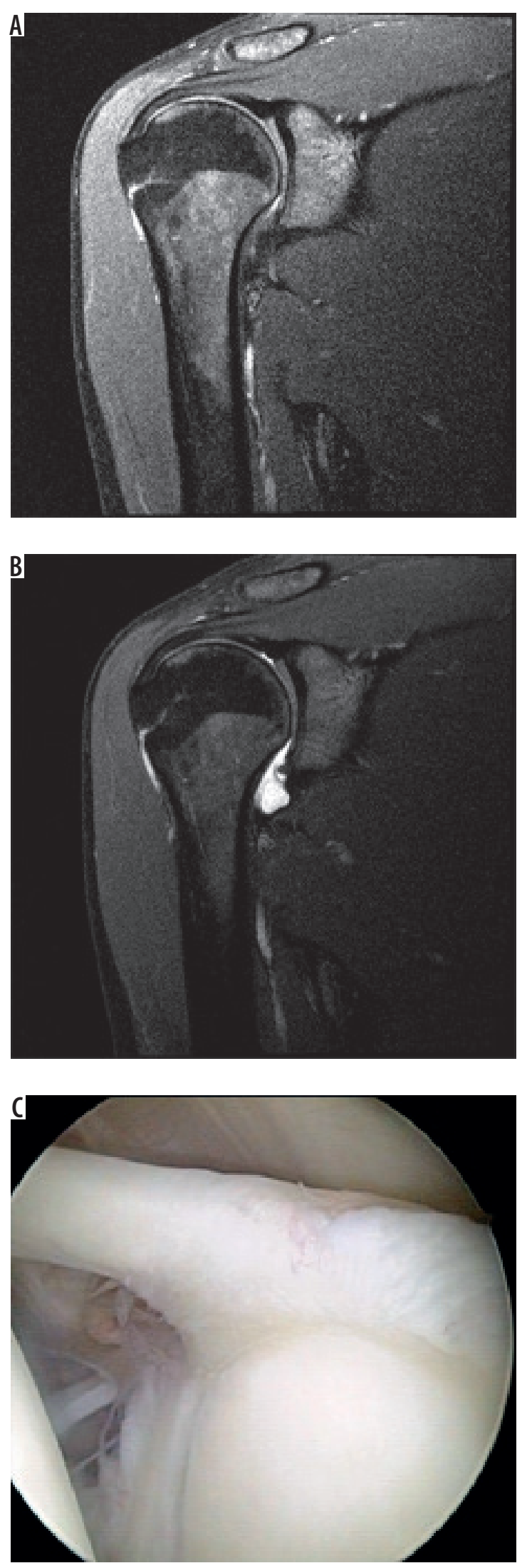

Figure 3. Normal superior capsulolabral complex. 30-year-old male athlete with a normal superior capsulolabral complex. Coronal fat-suppressed T2-weighted conventional magnetic resonance imaging (A) and magnetic resonance arthrogram images (B) show normal low signal intensity fibrocartilage; no contrast material extends into the labrum or into the chondrolabral junction. The corresponding arthroscopic evaluation (C) shows a normal superior capsulolabral complex
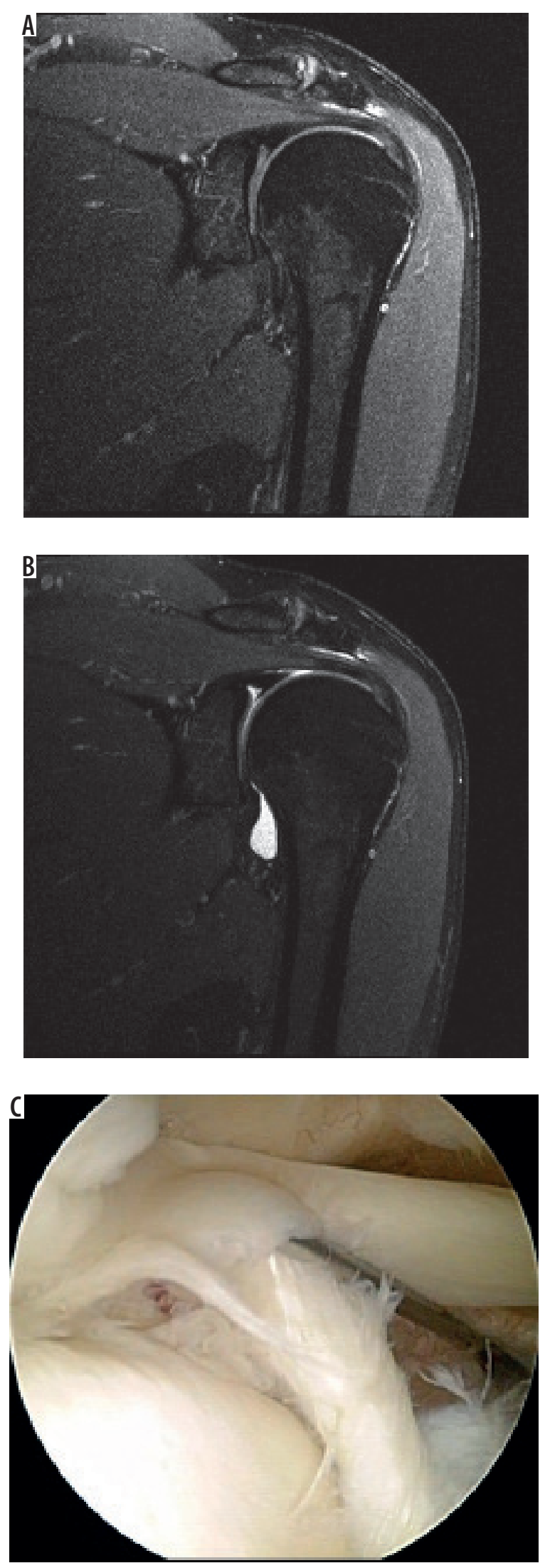

Figure 4. Superior labrum anterior-posterior (SLAP) lesion type II according to the Snyder classification. 39-year-old male athlete with a SLAP II lesion. Coronal fat-suppressed T2-weighted conventional magnetic resonance imaging $(A)$ and magnetic resonance arthrogram images (B) show a detachment with a laterally curved linear signal separating the superior labrum from the glenoid rim. The corresponding arthroscopic evaluation (C) reveals a SLAP lesion type II according to the Snyder classification 
gram images show normal low signal intensity fibrocartilage; no contrast material extends into the labrum or into the chondrolabral junction.

Figure 4 presents MRI and MDSA scans in coronary view showing a SLAP lesion $\mathrm{II}^{\circ}$ according to the Snyder classification and the corresponding arthroscopy finding.

\section{Discussion}

SLAP lesions of the shoulder are a common source of shoulder pain, and diagnosis by clinical means alone may be difficult. Hence, the radiological diagnosis of SLAP lesions based on different MRI and MRA techniques. However, regarding various methodical aspects and the heterogeneous evidence currently available in the literature focusing the diagnostic performance of MRA versus MRI to detect SLAP lesions, it remains controversial which is the most appropriate type of MR imaging for the diagnosis of SLAP tears. Therefore, this study investigated MDSA, a novel method of MRA technique considering the requirements of a practicable procedure in daily practice diagnosing suspected SLAP lesions, and compared the diagnostic findings with those of an established MRI investigation using the findings in arthroscopic surgery as a reference. The current study shows that diagnostic value the MDSA is significantly better than that of MRI examinations without included arthrography, and that the applied modified MRA provides the requirements as a practicable routine shoulder MR examination including arthrography for the detection of suspected suspicious SLAP lesions in a single examination.

The complex anatomy and wide range of traumatic and/or degenerative alterations are a major challenge in daily practice for an exact and adequate radiological diagnosis of the shoulder joint $[14,15]$. In particular, the superior capsulolabral complex as the origin of the long head biceps tendon shows a high variation in the physiological anatomy [16]. These variations are often of major importance for difficult differentiation between traumatic and degenerative pathologies. Furthermore, the exact discrimination of physiological variants from lesions of the superior capsulolabral complex have a high practical relevance: suitable therapy may differ in patients suffering from pain in disability with a clinically suspected biceps tendon pathology, for instance in athletes with different activity levels. Snyder et al. [3] and Handelberg [4] could demonstrate that alteration of the superior capsulolabral complex were still present in $6 \%$ of all shoulder arthroscopies with SLAP lesions type II in 55\% resp. 53\% as the most frequent type of SLAP pathologies. The same studies showing that SLAP lesions of type I were still present in $21 \%$ resp. $9.5 \%$ whereas SLAP lesions type III and IV were rarely found.

Currently, MRA offers the highest accuracy in detecting SLAP lesions $[10,11,17]$. The intra-articular application of contrast medium indirectly allows - through the distension of the superior capsulolabral complex as well as its specific impact by distribution in the joint - a better differentiation of the intra-articular structures and the identification of pathological changes. Based on different signal intensities around the superior capsulolabral complex, MRA leads to a better delineation of the structures in comparison to MRI, which is appropriate for the detection of pathologies and their differentiation from physiological variants such as a sublabral recess $[18,19]$.

The present study shows that the sensitivity $(p<0.001)$ and accuracy ( $p=0.001$ ) of MDSA are significantly higher compared to MRI in the detection of SLAP lesions, whereas there was no significant difference in specificity between both investigations $(p=0.625)$. Therefore, the results of the present study were in accordance with the findings of Magee, Bencardino, and Symanski, which also show a better accuracy of MRA compared to MRI in the detection of SLAP lesions $[10,11,17]$.

However, despite these data MRI is the dominate investigation in the detection of SLAP lesions until now in routine clinical practice, although the accuracy, with sensitivity about $14 \%$ and $63 \%$ and the specificity about $79 \%$ and $89 \%$, is inferior to MRA $[10,14,17,20-22]$. These may be related to some methodical aspects of the MRA as an invasive radiological investigation. Besides the associated higher cost and effort on a time and material basis, it is also associated by the potential risk of adverse reaction of the contrast medium and general complications of intraarticular injections.

The MDSA method, as described in this study, has the advantage that the methodical effort is considerably lower as compared to other established MRA techniques such as contrast medium application without imaging or with conventional fluoroscopy, sonography, or CT guidance [23-25].

The fluoroscopy-, sonography-, or CT-guided MRA investigations, besides the supply of the MR-unit, also require the availability and coordination of other radiological equipment. Furthermore, the required transport and preparation of the patients may result in longer time duration of the whole procedure. This prolonged time interval between contrast media application by different means and the origin MR imaging also have the potential disadvantage of reduced quality of the MR images due to the distribution and/or dilution of the contrast medium [26].

The overall mean duration of our approach of MRSA was 11 minutes and was comparable to other MRA techniques, e.g. fluoroscopy or sonography with the need of additional time for patient transport and positioning [24].

In the present study, an open 1.0-MRI scanner with a dedicated three-channel shoulder coil was used. Easier patient access referring to open construction of the MRI scanner enabled the direct manipulation of the patient. Therefore, two examinations - one with and one without contrast media - could be performed without the need of an additional change of the patient's position. This approach has the additional advantage that the risk of a sys- 
tematic mistake relating to the analysis of the MRI scans due to different positions of the patients is eliminated.

We are aware that field strength 3.0-T MR tomography is currently routinely used in most cases of suspected SLAP lesions. Despite the potentially better diagnostic accuracy due to the higher resolution regarding this field strength, there are still some methodical limitations that may influence the detection of these lesions. In this context, the position of the patient in the conventional closed 3.0-T MRI scanner in the middle of the table results in a more lateral positioning of the investigated shoulder compared to the centre of the magnetic field. The potential occurrence of inhomogeneities may result in reduced image quality. In contrast, the open 1.0-T MRI scanner used in this study enabled the positioning of the shoulder directly in the centre of the magnetic field. Furthermore, Magee et al. [11] showed that the sensitivity by detecting SLAP lesions using the 3.0-T MR was $83 \%$, whereas the sensitivity with MRA was $98 \%$ regardless of the mentioned field strength.

Also, a meta-analysis by Symanski et al. focussing the value of the MRI diagnostic of SLAP lesions demonstrated that sensitivity and specificity with 3.0-T MRI was 78.4-84.4\% and $95.2-98.8 \%$, respectively, and was significantly higher compared to those by $1.5-\mathrm{T}$ MRI at 79.1$81.2 \%$ and $66.7-83.6 \%$, respectively [17].

However, this study confirmed also that the accuracy of MRA was significantly higher in comparison with 3.0-T MRI (sensitivity $80.4 \%$ vs. $63.0 \%$ and specificity $90.7 \%$ vs. $87.2 \%)$.

A limitation of this study was the relative long interval of 77 days between the MR diagnosis and the arthroscopy. Therefore, it cannot be excluded that discrepancies be- tween the MR and arthroscopic diagnosis may be related to possible changes in the superior capsulolabral complex during this period; otherwise, the mentioned time interval reflects the daily clinical practice. For example, based on the MR diagnosis in some patients another non-operative treatment was attempted, or other individual reasons were responsible for this fact.

Another methodical aspect of this study was that the analysis of the MR interventions was performed by only a single radiologist who was very experienced in the MR diagnosis of musculoskeletal disorders, especially in shoulder pathologies. In this context, Grinsven et al. [27] showed that the diagnostic accuracy of the MR evaluation in patients with lesions of the capsulolabral complex strongly correlated with the experience of the radiological investigator and could also be significantly improved by consensus of more than one examiner. Otherwise, the latter conclusion is barely convertible, and the design of the present study better reflects daily clinical practice.

\section{Conclusions}

The MDSA with an open 1.0-Tesla MR scanner can be performed with high technical success as well as reasonable methodical use. For this reason, this modified MRA seems to be appropriate for implemention as a routine examination for SLAP lesions, mostly due to much higher diagnostic accuracy.

\section{Conflict of interest}

The authors declare no conflict of interest.

\section{References}

1. Mohana-Borges AV, Chung CB, Resnick D. Superior labral anteroposterior tear: classification and diagnosis on MRI and MR arthrography. AJR Am J Roentgenol 2003; 181: 1449-1462.

2. Andrews JR, Carson WG Jr., McLeod WD. Glenoid labrum tears related to the long head of the biceps. Am J Sports Med 1985; 13 337-341.

3. Snyder SJ, Karzel RP, Del PW, et al. SLAP lesions of the shoulder. Arthroscopy 1990; 6: 274-279.

4. Handelberg F, Willems S, Shahabpour M, et al. SLAP lesions: a retrospective multicenter study. Arthroscopy 1998; 14: 856-862.

5. Maffet MW, Gartsman GM, Moseley B. Superior labrum-biceps tendon complex lesions of the shoulder. Am J Sports Med 1995; 23: 93-98.

6. Lichtenberg S, Magosch P, Habermeyer P. Superior labrum-biceps anchor complex. Orthopade 2003; 32: 616-626.

7. Waldt S, Burkart A, Lange P, et al. Diagnostic performance of MR arthrography in the assessment of superior labral anteroposterior lesions of the shoulder. AJR Am J Roentgenol 2004; 182: 1271-1278.
8. Razmjou H, Fournier-Gosselin S, Christakis M, et al. Accuracy of magnetic resonance imaging in detecting biceps pathology in patients with rotator cuff disorders: comparison with arthroscopy. J Shoulder Elbow Surg 2016; 25: 38-44.

9. Wortler K, Waldt S, Burkhart A, et al. MR imaging of variants of the superior labral-bicipital complex and SLAP lesions. Orthopade 2003; 32: 595-599.

10. Bencardino JT, Beltran J, Rosenberg ZS, et al. Superior labrum anterior-posterior lesions: diagnosis with MR arthrography of the shoulder. Radiology 2000; 214: 267-271.

11. Magee T. 3-T MRI of the shoulder: is MR arthrography necessary? AJR Am J Roentgenol 2009; 192: 86-92.

12. Major NM, Browne J, Domzalski T, et al. Evaluation of the glenoid labrum with 3-T MRI: is intraarticular contrast necessary? AJR Am J Roentgenol 2011; 196: 1139-1144.

13. Wybranski C, Adamchic I, Rohl FW, et al. Freehand direct arthrography of the shoulder using near real-time guidance in an open 1.0-T MRI scanner. Skeletal Radiol 2017; 46: 51-58. 
14. Sheridan K, Kreulen C, Kim S, et al. Accuracy of magnetic resonance imaging to diagnose superior labrum anterior-posterior tears. Knee Surg Sports Traumatol Arthrosc 2015; 23: 2645-2650.

15. Amin MF, Youssef AO. The diagnostic value of magnetic resonance arthrography of the shoulder in detection and grading of SLAP lesions: comparison with arthroscopic findings. Eur J Radiol 2012; 81: 2343-2347.

16. Chang D, Mohana-Borges A, Borso M, et al. SLAP lesions: anatomy, clinical presentation, MR imaging diagnosis and characterization. Eur J Radiol 2008; 68: 72-87.

17. Symanski JS, Subhas N, Babb J, et al. Diagnosis of superior labrum anterior-to-posterior tears by using $\mathrm{mr}$ imaging and $\mathrm{mr}$ arthrography: a systematic review and meta-analysis. Radiology 2017; 285: 101-113.

18. Jin W, Ryu KN, Kwon SH, et al. MR arthrography in the differential diagnosis of type II superior labral anteroposterior lesion and sublabral recess. AJR Am J Roentgenol 2006; 187: 887-893.

19. Modarresi S, Motamedi D, Jude CM. Superior labral anteroposterior lesions of the shoulder: part 1, anatomy and anatomic variants. AJR Am J Roentgenol 2011; 197: 596-603.

20. Connolly KP, Schwartzberg RS, Reuss B, et al. Sensitivity and specificity of noncontrast magnetic resonance imaging reports in the diagnosis of type-II superior labral anterior-posterior lesions in the community setting. J Bone Joint Surg Am 2013; 95: 308-313.
21. Connell DA, Potter HG. Magnetic resonance evaluation of the labral capsular ligamentous complex: a pictorial review. Australas Radiol 1999; 43: 419-426.

22. Phillips JC, Cook C, Beaty S, et al. Validity of noncontrast magnetic resonance imaging in diagnosing superior labrum anterior-posterior tears. J Shoulder Elbow Surg 2013; 22: 3-8.

23. Powell SE, Davis SM, Lee EH, et al. Accuracy of palpation-directed intra-articular glenohumeral injection confirmed by magnetic resonance arthrography. Arthroscopy 2015; 31: 205-208.

24. Ng AW, Hung EH, Griffith JF, et al. Comparison of ultrasound versus fluoroscopic guided rotator cuff interval approach for MR arthrography. Clin Imaging 2013; 37: 548-553.

25. Mulligan ME. CT-guided shoulder arthrography at the rotator cuff interval. AJR Am J Roentgenol 2008; 191: W58-W61.

26. Andreisek G, Duc SR, Froehlich JM, et al. MR arthrography of the shoulder, hip, and wrist: evaluation of contrast dynamics and image quality with increasing injection-to-imaging time. AJR Am J Roentgenol 2007; 188: 1081-1088.

27. van GS, Hagenmaier F, van Loon CJ, et al. Does the experience level of the radiologist, assessment in consensus, or the addition of the abduction and external rotation view improve the diagnostic reproducibility and accuracy of MRA of the shoulder? Clin Radiol 2014; 69: 1157-1164. 